\title{
Design principles of sensory receptors
}

\author{
Dieter Wicher* \\ Laboratory of Neurophysiology, Department of Evolutionary Neuroethology, Max Planck Institute for Chemical Ecology, Jena, Germany \\ ${ }^{*}$ Correspondence: dwicher@ice.mpg.de
}

Organisms continuously detect and process physical and chemical signals from their external and internal world, and they monitor their interaction with the environment. Aristotle was the first who defined the five external senses in humans: sight, hearing, smell, taste, and touch. In addition, we consider balance as the sixth external sense. Similarly important for the control of movement is the sense of body position, the proprioception. The physiological state of the organism is reported by a variety of internal receptors including those for gases, temperature, or $\mathrm{pH}$. According to the activating stimulus sensory receptors can be classified into electromagnetic receptors (photoreceptor, thermoreceptor), mechanoreceptors (hearing, touch, balance, osmoreceptor), and chemoreceptors (odorant receptor, gustatory receptor). Sensory signals are perceived by specialized neurons equipped with one type of receptor molecules as photoreceptor cells or with various types of receptors as nociceptive neurons to detect different noxious stimuli including heat, pressure, $\mathrm{pH}$, or chemical signals. Most receptor molecules are tuned to a single sensory modality but some are polymodal as the vanilloid receptor VR1 which is activated by heat, pungent chemicals, acids, or lipids. Activation of receptor molecules by an adequate stimulus initiates a signal transduction process in the sensory neuron in which the physical or chemical signal is amplified and converted into an electrical signal that depolarizes or hyperpolarizes the cell. The properties of the stimulus such as strength and duration are then translated into a specific temporal pattern of action potentials which is further processed in the brain.

In recent years the research on design and function of sensory receptors and their signal transduction machinery has largely extended our knowledge in this field. The present special issue is aimed of presenting an actual view on photoreceptors and chemoreceptors.

Photoreceptors and many chemoreceptors belong to the $G$ protein-coupled receptor (GPCR) family. GPCRs are seven transmembrane (7-TM)-spanning proteins that interact with heterotrimeric G proteins. According to structural similarities GPCRs are grouped into families. Photoreceptors and most vertebrate olfactory receptors belong to the rhodopsin receptor/class A family. Ligand binding to a GPCR causes a conformational change thereby activating a trimeric $G$ protein which disensembles into the $\mathrm{G}_{\alpha}$ - and the $\mathrm{G}_{\beta \gamma}$-subunit. Independently, both subunits can affect various targets such as enzymes or ion channels. G protein signaling is usually associated with signal amplification. An exception are mammalian olfactory receptors in which the dwell time of odorant molecules is too short to efficiently activate $\mathrm{G}$ proteins (Bhandawat et al., 2005). The signal amplification takes place downstream by interaction of ion channels.

In the classical view a monomeric protein with 7-TM topology constitutes a metabotropic receptor (Figure 1A) which interacts with a trimeric $\mathrm{G}$ protein. However, in the case of the channelrhodopsins the 7-TM protein does not couple to a G protein. Instead, it functions as an ionotropic receptor (Figure 1B) since its activation gates an ion channel (Nagel et al., 2005). GPCRs may form homomeric (Figure 1C) as well as heteromeric dimers (Figure 1D). For class A GPCRs instable homodimerization has been observed for M1 muscarinic acetylcholine receptors (Hern et al., 2010) or an oligomerization to higher orders as for $\beta 2$-adrenergic receptors (Fung et al., 2009). Class C GPCRs built stable heterodimers such as the mammalian taste receptors for sweet and umami (Palmer, 2007). In heterodimeric $\mathrm{GABA}_{\mathrm{B}}$ receptors, $\mathrm{GABA}_{\mathrm{B}} \mathrm{R} 1$ binds the ligand while $G_{A B A_{B}} R 2$ couples to the $G$ protein and is required for targeting $\mathrm{GABA}_{\mathrm{B}} \mathrm{R} 1$ to the membrane (Brauner-Osborne et al., 2007). Intriguingly, a similar principle is observed in insect odorant receptors. These 7-TM proteins are heterodimers of a conventional odorant receptor (OR) protein and an ubiquitous co-receptor (Neuhaus et al., 2005) (Figure 1E). The OR protein binds the odorant and couples to the $G$ protein which in turn stimulates cAMP production (Wicher et al., 2008). The co-receptor is required for membrane targeting of the OR protein (Larsson et al., 2004) and is a non-selective cation channel activated by cAMP (Wicher et al., 2008). In addition to this metabotropic pathway of insect OR activation there is also a $\mathrm{G}$ protein independent, ionotropic one (Sato et al., 2008; Wicher et al., 2008). These findings raised a controversial discussion how to classify insect ORs, as ionotropic or metabotropic. One view is "In insects, most odorant receptors consist of a heteromeric complex that serves both as the receptor for the ligand and as the ion channel that is gated by binding of the ligand - a mechanism that is referred to as ionotropic" (Kaupp, 2010). The other view is "The design principle of insect ORs of being composed of an odorant-sensitive protein and of a protein finally transducing the chemical message into an electrical signal ensures very rapid recognition of high odor concentrations via the ionotropic pathway as well as a somewhat slower but prolonged and highly sensitive odor detection via the G protein-mediated signal amplification" (Wicher et al., 2008). Per definition an ionotropic receptor is an ion channel directly gated by ligand binding. This is not the case for insect ORs at low odor concentration where channel opening requires $G$ protein activation and second messenger production - hallmarks of metabotropic signaling. This kind of signal transduction is quite similar to the olfactory signaling in vertebrates. The only difference is that in vertebrates the activated ion channel is separated from the receptor while in insects it is part of the receptor. At high odor concentration the metabotropic pathway is accompanied and sped up by the ionotropic one. Thus, a more appropriate classification of insect ORs might be "combined metabotropic and ionotropic receptors". A detailed discussion on insect ORs is given by $\mathrm{Ha}$ and Smith.

In Drosophila photoreceptors the signal transduction machinery is highly organized. Their elements are held together by the 


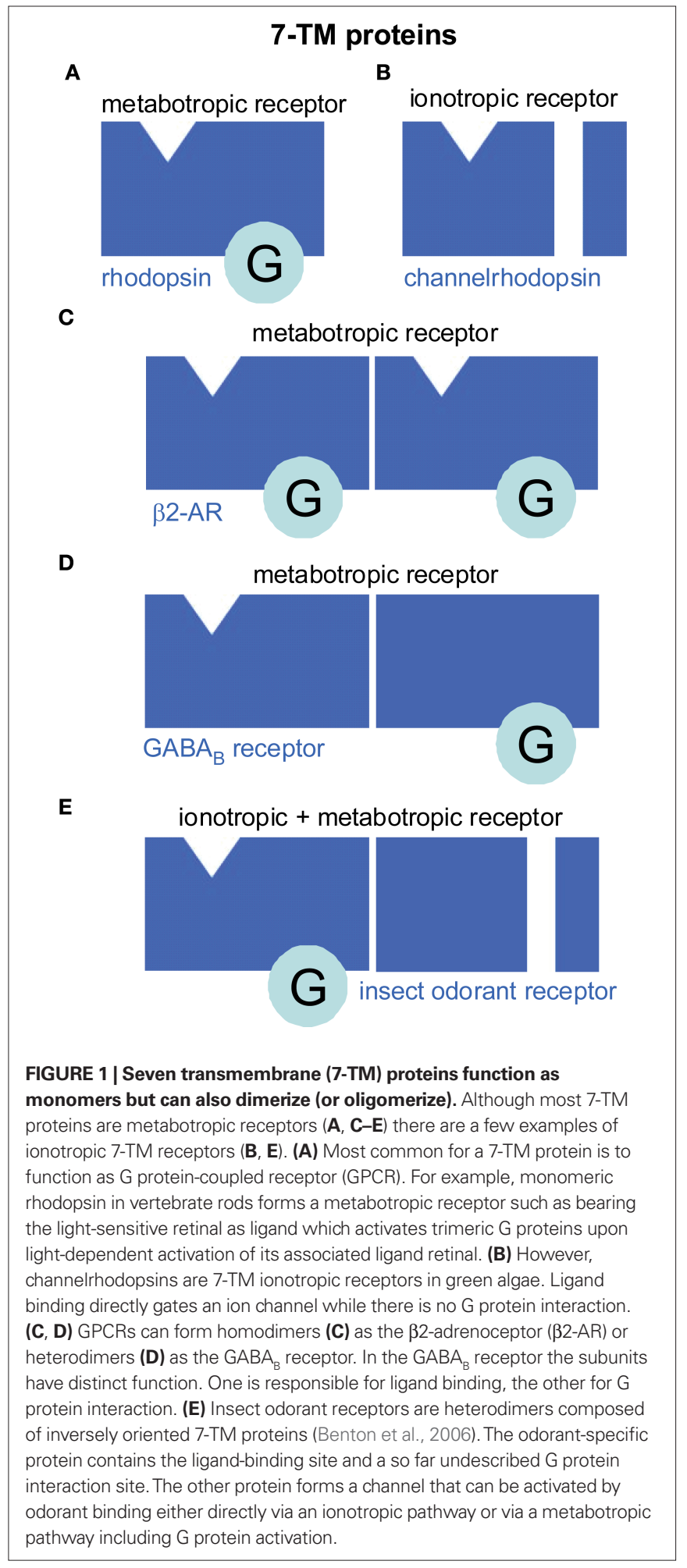

scaffolding protein InaD which enables fast processing of the sensory input. The delay between photon absorption by rhodopsin and activation of the receptor current is only $20 \mathrm{~ms}$. The ion channel conducting this current was the first member of the TRP channel superfamily to be detected. Mutant flies with disrupted channel function show the phenotype of Transient Receptor Potential upon constant light stimulus (Cosens and Manning, 1969). Various members of the TRP superfamily form sensory receptors including mechanoreceptors, thermoreceptors, chemoreceptors, and pain receptors (Damann et al., 2008), some of which are polymodal (Belmonte and Viana, 2008). Katz and Minke review anatomy and function of fly photoreceptors and provide a current view on details of sensory transduction.

Retinal ganglion cells expressing melanopsin are photosensitive cells that do not contribute to image-processing vision but are involved in the entrainment of circadian rhythms. GonzalezMenendez et al. demonstrate in their research report that melanopsin-expressing cells themselves are subjected to circadian regulation. They found a circadian oscillation of the number of these cells in the mouse retina which required synchronization by the light/ dark cycle.

A review related to non-visual photoreception is presented by Gotow and Nishi on photosensitive interneurons modulating the behavior of a sea slug. These neurons are localized in central ganglia and respond to light either with depolarizations or with hyperpolarizations. The type of the photoresponse depends on coupling to G proteins which lead either to cGMP hydrolysis or synthesis subsequently closing or opening a cGMP-dependent $\mathrm{K}^{+}$ channel, respectively.

Chemoreceptors are another class of sensory receptors using GPCRs - though not exclusively - to detect chemical signaling molecules. An update on mammalian olfactory receptors is given by Fleischer et al. According to structure and distribution these receptor proteins are grouped into families including ORs, vomeronasal receptors (VRs), trace amine-associated receptors (TAARs), formyl peptide receptors (FPRs), and guanylyl cylase D (GCD). Except for the GCDs all other receptors form GPCRs. ORs, TAARs and the VR1 subgroup belong to the rhodopsin/class A family. The VR2 subgroup belongs to the class $\mathrm{C}$ family, whether their members dimerize remains to be shown.

Insect ORs, as reviewed by $\mathrm{Ha}$ and Smith, differ from classical GPCRs in various aspects. Although the receptor proteins have a 7-TM structure like GPCRs they do not share sequence similarity with them. In addition, insect odorant receptor proteins show an inverted orientation in the membrane, i.e., the $\mathrm{N}$-terminus is intracellular and the C-terminus extracellular. Finally, these ORs are heterodimers composed of a conventional, odor-specific receptor protein and an ubiquitous protein such as Or83b in Drosophila (Figure 1E). There is a distinct class of putative chemosensory receptors that are akin to ionotropic glutamate receptors. Drosophila pheromone receptors are composed of a conventional OR and Or83b. But in contrast to ORs another, receptor-associated sensory neuron membrane protein (SNMP) with 2-TM topology is required to form a functional complex. Intriguingly, it is SNMP that binds the ligand which is not the pheromone itself but the pheromone-binding protein LUSH when activated by the pheromone.

The review by Stengl on moth pheromone receptors demonstrates how the high sensitivity of male moth pheromone receptor neurons is obtained which is necessary to perceive the intermittently 
released sex-pheromone blend. It becomes apparent that depending on the stimulus properties different signal transduction pathways are recruited. Furthermore, the sensitivity of receptor neurons is regulated in a circadian manner to achieve highest sensitivity during the moth's activity phase and to allow for a phase of rest.

The contribution by Isono and Morita reviews the present knowledge on insect gustatory receptors (GRs) which are distantly related to the insect ORs. While in the olfactory system one neuron expresses one receptor, various GRs may be expressed in taste neurons. Moreover, these neurons also express other sensory receptors such as the TPR channel painless. This principle allows one neuron to perceive different sensory modalities.

\section{REFERENCES}

Belmonte, C., and Viana, F. (2008). Molecular and cellular limits to somatosensory specificity. Mol. Pain 4,14 .

Benton, R., Sachse, S., Michnick, S. W., and Vosshall, L. B. (2006). Atypical membrane topology and heteromeric function of Drosophila odorant receptors in vivo. PLoS Biol. 4, e20. doi:10.1371/ journal.pbio.0040020.

Bhandawat, V., Reisert, J., and Yau, K. W. (2005). Elementary response of olfactory receptor neurons to odorants. Science 308, 1931-1934.

Brauner-Osborne, H., Wellendorph, P., and Jensen, A. A. (2007). Structure, pharmacology and therapeutic prospects of family C G-protein coupled receptors. Curr. Drug Targets 8, 169-184.

Cosens, D. J., and Manning, A. (1969). Abnormal electroretinogram from a Drosophila mutant. Nature 224, 285-287.

Damann, N., Voets, T., and Nilius, B. (2008). TRPs in our senses. Curr. Biol. 18, R880-R889.

Fung, J. J., Deupi, X., Pardo, L., Yao, X. J., R. K., and Kobilka, B. K. (2009). Ligandregulated oligomerization of beta(2)adrenoceptors in a model lipid bilayer. EMBO J. 28, 3315-3328.

Hern, J. A., Baig, A. H., Mashanov, G. I., Birdsall, B., Corrie, J. E., Lazareno, S., Molloy, J. E., and Birdsall, N. J. (2010). Formation and dissociation of M1 muscarinic receptor dimers seen by total internal reflection fluorescence imaging of single molecules. Proc. Natl. Acad. Sci. U.S.A. 107, 2693-2698.

Kaupp, U. B. (2010). Olfactory signalling in vertebrates and insects: differences and commonalities. Nat. Rev. Neurosci. $11,188-200$ Velez-Ruiz, G.A.,Devree, B.T.,Sunahara,

The function of GPCRs and downstream signaling is subject to extensive modulation, for example by RGS proteins (regulator of $\mathrm{G}$ protein signaling). Fenech et al. present an original article on Ric-8A, a guanine nucleotide exchange factor. This $\mathrm{G}$ protein interacting protein is localized in taste receptor cells and is able to amplify the response to a taste signal by promoting the exchange of GDP by GTP.

This special issue comprises various examples how the receptor design is adapted to the physiological demands of an organism to perceive sensory signals in different intensity and temporal resolution. There is accumulating evidence that often a sensory receptor is part of a signal processing network including scaffolding proteins or RGS proteins.

Larsson, M. C., Domingos, A. I., Jones, W. D., Chiappe, M. E., Amrein, H., and Vosshall, L. B. (2004). Or83b encodes a broadly expressed odorant receptor essential for Drosophila olfaction. Neuron 43, 703-714

Nagel, G., Szellas, T., Kateriya, S.,Adeishvili, N., Hegemann, P., and Bamberg, E. (2005). Channelrhodopsins: directly light-gated cation channels. Biochem. Soc. Trans. 33, 863-866.

Neuhaus, E. M., Gisselmann, G., Zhang, W., Dooley, R., Störtkuhl, K., and Hatt, H. (2005). Odorant receptor heterodimerization in the olfactory system of Drosophila melanogaster. Nat. Neurosci. 8, 15-17.

Palmer, R. K. (2007). The pharmacology and signaling of bitter, sweet, and umami taste sensing. Mol. Interv. 7, 87-98.

Sato, K., Pellegrino, M., Nakagawa, T. Nakagawa, T., Vosshall, L.B., and
Touhara, K. (2008). Insect olfactory receptors are heteromeric ligand-gated ion channels. Nature 452, 1002-1006.

Wicher, D., Schäfer, R., Bauernfeind, R., Stensmyr, M. C., Heller, R., Heinemann, S.H., and Hansson, B. S. (2008). Drosophila odorant receptors are both ligand-gated and cyclicnucleotide-activated cation channels. Nature 452, 1007-1011.

Received: 02 June 2010; accepted: 15 June 2010; published online: 07 July 2010.

Citation: Wicher D (2010) Design principles of sensory receptors. Front. Cell. Neurosci. 4:25. doi: 10.3389/fncel.2010.00025 Copyright (c) 2010 Wicher. This is an openaccess article subject to an exclusive license agreement between the authors and the Frontiers Research Foundation, which permits unrestricted use, distribution, and reproduction in any medium, provided the original authors and source are credited. 